\title{
90.
}

\section{NOTE SUR QUELQUES FORMULES RELATIVES AUX CONIQUES.}

[From the Journal für die reine und angewandte Mathematik (Crelle), tom. xxxıx.

(1850), pp. 1-3.]

SoIT, comme à l'ordinaire :

$$
\left.\begin{array}{l}
\mathfrak{A}=B C-F^{2}, \\
\mathfrak{a}=C A-G^{2}, \\
\mathfrak{C}=A B-H^{2}, \\
\sqrt[f]{\mathfrak{F}}=G H-A F, \\
\boldsymbol{G}=H F-B G, \\
\boldsymbol{G}=F G-C H, \\
K=A B C-A F^{2}-B G^{2}-C H^{2}+2 F G H,
\end{array}\right\}
$$

et désignons, pour abréger, les fonctions

$$
\begin{aligned}
& A x^{2}+B y^{2}+C z^{2}+2 F y z+2 G x z+2 H x y, \\
& A \alpha x+B \beta y+C \gamma z+F(\beta z+\gamma y)+G(\gamma x+\alpha z)+H(\alpha y+\beta x) \text {, } \\
& A(\beta z-\gamma y)^{2}+B(\gamma x-\alpha z)^{2}+C(\alpha y-\beta x)^{2}+2 F(\gamma x-\alpha z)(\alpha y-\beta x) \\
& \text { \&c. } \\
& +2 G(\alpha y-\beta x)(\beta z-\gamma y)+2 H(\beta z-\gamma y)(\gamma x-\alpha z) \text {; }
\end{aligned}
$$

par

$$
A x^{2}+\ldots ; A \alpha x+\ldots ; A(\beta z-\gamma y)^{2}+\ldots ; \& c .
$$

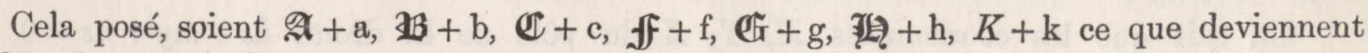

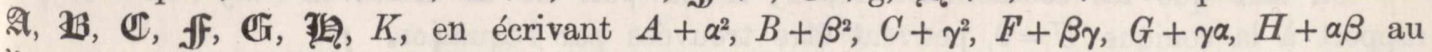
lieu de $A, \stackrel{B}{B} C, F, G, H$. Alors on aura d'abord'

$$
\mathrm{k}=\mathfrak{A} \alpha^{2}+\ldots
$$


et les quantités $\mathrm{a}, \mathrm{b}, \mathrm{c}, \mathrm{f}, \mathrm{g}, \mathrm{h}$ seront données par l'équation

$$
\mathrm{a} x^{2}+\ldots=A(\beta z-\gamma y)^{2}+\ldots
$$

ou, si l'on veut, par celle-ci :

$$
\operatorname{axx} x_{1}+\ldots=A(\beta z-\gamma y)\left(\beta z_{1}-\gamma y_{1}\right)+\ldots
$$

(savoir, en considérant ces équations comme identiques par rapport à $x, y, z$ et $x_{1}, y_{1}, z_{1}$ respectivement).

On obtient sans difficulté les équations identiques:

$$
\begin{aligned}
& \left(A \alpha \alpha_{1}+\ldots\right)\left(A x x_{1}+\ldots\right)-(A \alpha x+\ldots)\left(A \alpha_{1} x_{1}+\ldots\right)=\mathbb{A}\left(\beta z_{1}-\gamma y_{1}\right)\left(\beta_{1} z-\gamma_{1} y\right)+\ldots \\
& \left(\mathfrak{A} \alpha \alpha_{1}+\ldots\right)\left(\mathfrak{A} x x_{1}+\ldots\right)-(\mathfrak{A} \alpha x+\ldots)\left(\mathfrak{A} \alpha_{1} x_{1}+\ldots\right)=K\left[A\left(\beta z_{1}-\gamma y_{1}\right)\left(\beta_{1} z-\gamma_{1} y\right)+\ldots\right]
\end{aligned}
$$

Comme on sait, la condition sous laquelle la droite $l x+m y+n z=0$ touche la conique $U=A x^{2}+\ldots=0$ peut être présentée sous la forme $\mathfrak{A} l^{2}+\ldots=0$. Donc la condition pour que cette droite touche la conique $U+(\alpha x+\beta y+\gamma z)^{2}=0$, est

$$
\mathfrak{A} l^{2}+\ldots+A(\gamma m-\beta n)^{2}+\ldots=0
$$

En réduisant au moyen de l'équation $\left(\mathfrak{A} \alpha^{2}+\ldots\right)\left(\mathfrak{A} l^{2}+\ldots\right)=K\left[A(\gamma m-\beta n)^{2}+\ldots\right]$ (laquelle n'est qu'un cas particulier de l'équation (6)), cette condition devient:

$$
\left(K+\mathfrak{A} \alpha^{2}+\ldots\right)\left(\mathfrak{A} l^{2}+\ldots\right)-(\mathfrak{A} \alpha l+\ldots)^{2}=0
$$

Pour trouver la condition sous laquelle les coniques

$$
U+(\alpha x+\beta y+\gamma z)^{2}=0, \quad U+\left(\alpha_{1} x+\beta_{1} y+\gamma_{1} z\right)^{2}=0
$$

se touchent, on n'a qu'à remarquer que l'équation de la tangente commune est, ou

$$
\left(\alpha-\alpha_{1}\right) x+\left(\beta-\beta_{1}\right) y+\left(\gamma-\gamma_{1}\right) z=0, \quad \text { ou } \quad\left(\alpha+\alpha_{1}\right) x+\left(\beta+\beta_{1}\right) y+\left(\gamma+\gamma_{1}\right) z=0 .
$$

En ne considérant que la première de ces droites, on a pour la condition cherchée :

$$
\mathfrak{A}\left(\alpha-\alpha_{1}\right)^{2}+\ldots+A\left(\beta \gamma_{1}-\beta_{1} \gamma\right)^{2}+\ldots=0
$$

ou, en réduisant au moyen du même cas particulier de l'équation (6), on obtient cette condition sous la forme

$$
\left(K+\mathfrak{A} \alpha^{2}+\ldots\right)\left(K+\mathfrak{A} \alpha_{1}^{2}+\ldots\right)-\left(K+\mathfrak{A} \alpha \alpha_{1}+\ldots\right)^{2}=0 .
$$

On sait que les coordonnées $X, Y, Z$ du pôle de la droite $l x+m y+n z=0$, par rapport à la conique $U=0$, peuvent être trouvées par l'équation

$$
\lambda X+\mu Y+\nu Z=\mathfrak{A} l \lambda+\ldots,
$$


(considérée comme identique par rapport à $\lambda, \mu, \nu$ ). De là les coordonnées $X, Y, Z$ du pôle de cette même droite par rapport à la conique

$$
U+(\alpha x+\beta y+\gamma z)^{2}=0
$$

se trouveront par l'équation

$$
\lambda X+\mu Y+\nu Z=\mathbb{A} l \lambda+\ldots+A(\gamma m-\beta n)(\gamma \mu-\beta \nu) \ldots
$$

(considérée comme identique par rapport à $\lambda, \mu, \nu$ ). Cette équation peut aussi être présentée sous la forme

$$
K(\lambda X+\mu Y+\nu Z)=\left(K+\mathfrak{A} \alpha^{2}+\ldots\right)(\mathfrak{A} l \lambda+\ldots)-(\mathfrak{A} \alpha \lambda+\ldots)(\mathfrak{A} \alpha l+\ldots) \quad \ldots(12),
$$

ce qui peut être démontré facilement au moyen d'un cas particulier de l'équation (6).

Si les deux droites $l x+m y+n z=0, l^{\prime} x+m^{\prime} y+n^{\prime} z=0$, touchent la conique $U=0$, l'équation de la droite qui passe par les' points de contact sera $A\left(m n^{\prime}-m^{\prime} n\right) x+\ldots=0$. Donc: si ces deux droites touchent la conique $U+(\alpha x+\beta y+\gamma z)^{2}=0$, l'équation de la droite qui passe par les deux points de contact sera

$$
A\left(m n^{\prime}-m^{\prime} n\right) x+\ldots+(\alpha x+\beta y+\gamma z)\left[\alpha\left(m n^{\prime}-m^{\prime} n\right)+\beta\left(n l^{\prime}-n^{\prime} l\right)+\gamma\left(l m^{\prime}-l^{\prime} m\right)\right]=0 \ldots
$$

Les formules obtenues seront utiles pour la solution du problème du mémoire suivant, [91]. Je les ai rapprochées ici pour ne pas interrompre cette solution. 